\title{
Spectrum of intracranial complications of rhino-orbito-cerebral mucormycosis - resurgence in the era of COVID-19 pandemic: a pictorial essay
}

\author{
Kavya S. Kaushik ${ }^{1}$ - Rupa Ananthasivan ${ }^{1}$ - Ullas V. Acharya ${ }^{1}$. Sudarshan Rawat ${ }^{1}$ - Uday Damodar Patil ${ }^{1}$. \\ Balasubramanyam Shankar ${ }^{1} \cdot$ Abin Jose $^{1}$
}

Received: 12 July 2021 / Accepted: 10 September 2021 / Published online: 4 October 2021

(c) American Society of Emergency Radiology 2021

\begin{abstract}
Rhino-orbito-cerebral mucormycosis (ROCM) has regained significance following its resurgence in the second wave of the COVID-19 pandemic in India. Rapid and progressive intracranial spread occurs either by direct extension across the neural foraminae, cribriform plate/ethmoid, walls of sinuses, or angioinvasion. Having known to have a high mortality rate, especially with intracranial extension of disease, it becomes imperative to familiarise oneself with its imaging features. MRI is the imaging modality of choice. This pictorial essay aims to depict and detail the various intracranial complications of mucormycosis and to serve as a broad checklist of structures and pathologies that must be looked for in a known or suspected case of ROCM.
\end{abstract}

Keywords ROCM $\cdot$ Mucormycosis · COVID-19 · Intracranial · Cavernous sinus thrombosis $\cdot$ Ischaemic neuropathy

Mucormycosis is an invasive, potentially fatal, opportunistic fungal infection, caused by saprophytic fungi of the order Mucorales. A rapid surge in its incidence has been witnessed in the second wave of the COVID-19 pandemic in India. The proposed predisposing factors include hypoxia, uncontrolled blood sugars either steroid induced or due to diabetes mellitus, and prolonged multipronged immunosuppression [1]. Though it can involve different organ systems, the most common type is rhino-orbitocerebral mucormycosis (ROCM) [2].

The route of infection is usually by inhalation of the spores which invade the mucosa of nasal cavity and sinuses causing rhinosinusitis. Further rapid and progressive intracranial spread of the fungus occurs either by direct extension across the neural foraminae, cribriform plate/ethmoid, walls of the frontal and sphenoid sinuses, or angioinvasion of the walls of the arteries and veins, causing vascular thrombosis, occlusion, and infarction [3-5].

Rupa Ananthasivan

rupanth@yahoo.com

1 Department of Radiology, Manipal Hospitals, 98, HAL Old Airport Road, Kodihalli, Bengaluru 560017, India
Symptoms of intracranial extension include altered sensorium, diplopia, ophthalmoplegia, cranial nerve deficits, and focal neurological deficits based on the region of spread. With the intracranial extension of disease, mortality is greater than $80 \%$ [3].

Early imaging is crucial and aids in assessing the extent of involvement of disease and assists in prompt initiation aggressive antifungal treatment and surgical debridement. Magnetic resonance imaging is the imaging modality of choice. Restricted diffusion in ROCM indicates tissue ischaemia and necrosis resulting from the angioinvasive property of the fungus and thereby the path of spread of fungal infection [6].

This pictorial review will showcase the MR imaging features of intracranial complications of ROCM. The commonest intracranial manifestations are cavernous sinus extension, narrowing or occlusion of the internal carotid artery (ICA), and infarction [7].

\section{Extension into cavernous sinus}

Cavernous sinus involvement can occur through the ethmoidal and orbital veins that drain into it, through direct extension from sphenoid sinus, or from perineural spread along neural foraminae [3-5]. Bulging of the 


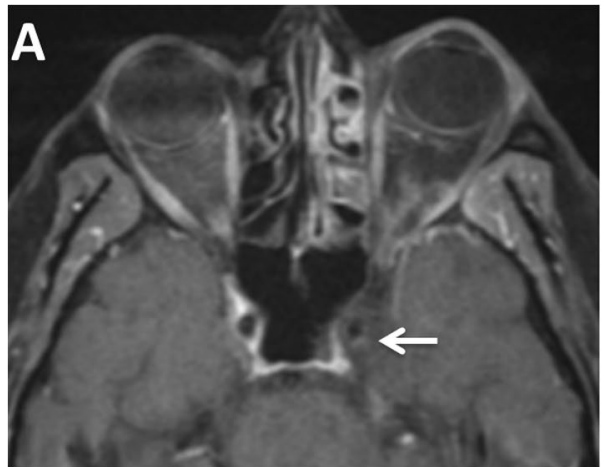

Fig. 1 Cavernous sinus thrombosis: A Axial post contrast T1-FSweighted image of the brain reveals non enhancement of the left cavernous sinus (white arrow), indicating cavernous sinus thrombosis. Normal enhancement of the right cavernous sinus is seen. Bilateral

lateral walls of the sinus, abnormal dural enhancement of the lateral wall, abnormal signal intensity of the sinus (iso-hypointensity on T1 and hyperintensity on T2), and

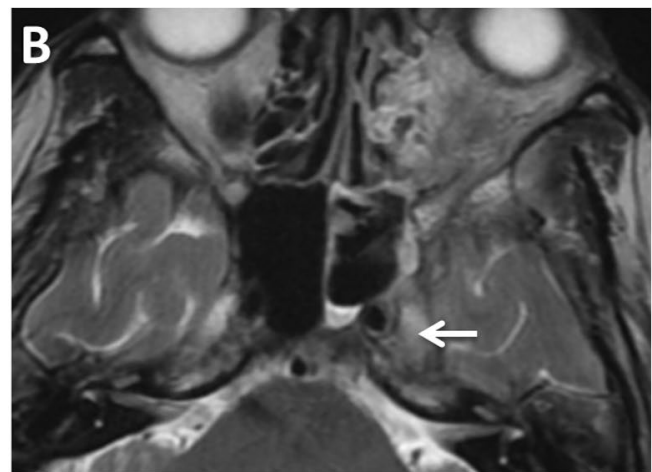

ICA are patent. B Axial T2-weighted image of the brain reveals T2 heterogeneously hyperintense left paracavernous soft tissue (white arrow) with convex bulge of the wall of the cavernous sinus on the left

presence of filling defects on post contrast scans (Fig. 1) indicate cavernous sinus thrombosis [8, 9]. Paracavernous soft tissue extension can also be seen (Figs. 1 and
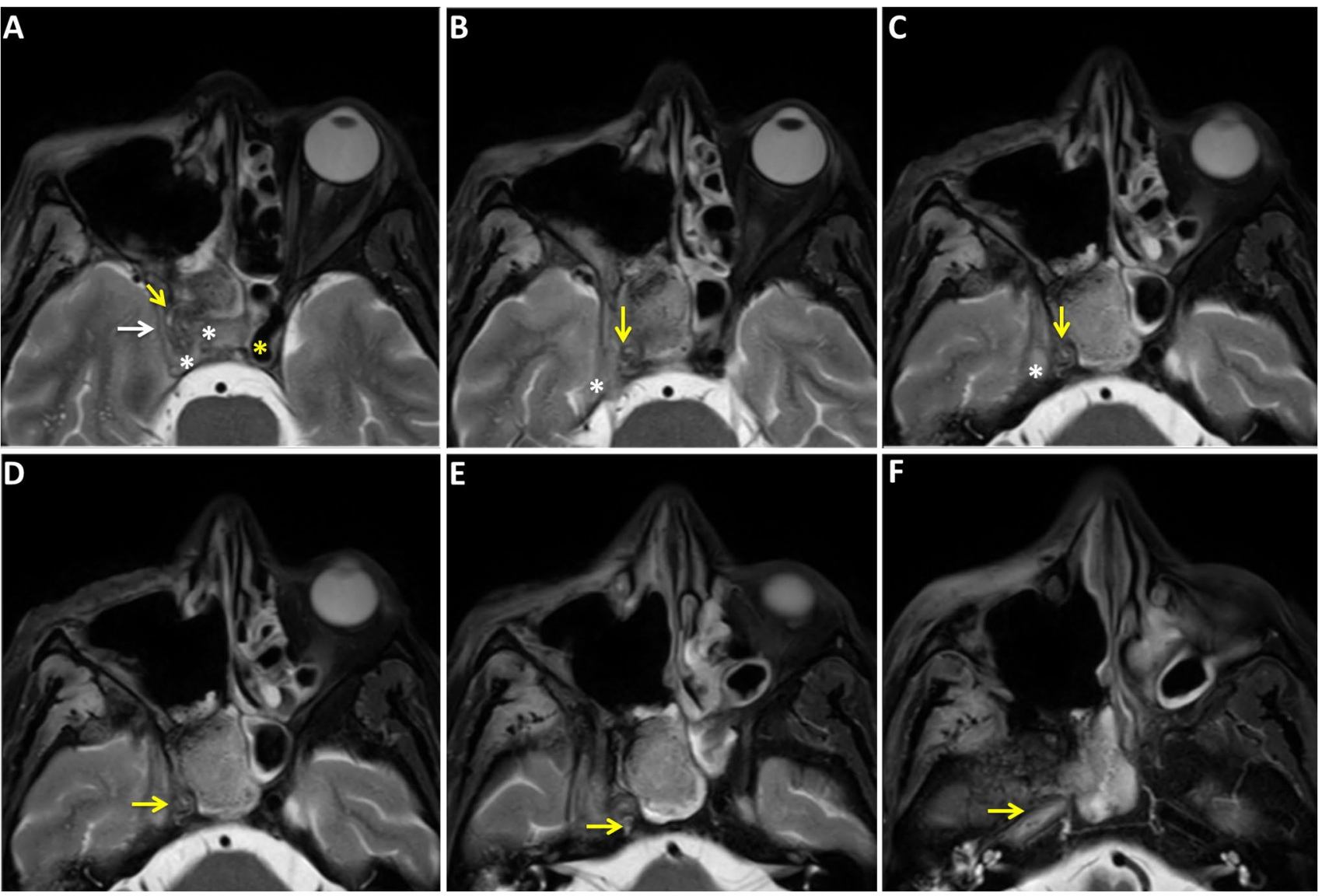

Fig. 2 Cavernous sinus extension and right ICA thrombosis: Axial T2-weighted images of the brain reveal A convex bulge of the wall of the cavernous sinus on the right (white arrow), as opposed to a normal convex wall as seen on the left; A T2 hyperintense soft tissue in the right cavernous sinus (white asterisk), $\mathbf{B}, \mathbf{C}$ extending into the right paracavernous region (white asterisk). A-F Invasion of the right ICA with resultant thrombosis is seen as loss of flow void in the petrocavernous right ICA (yellow arrows). Left intracranial ICA shows normal flow void (yellow asterisk) 


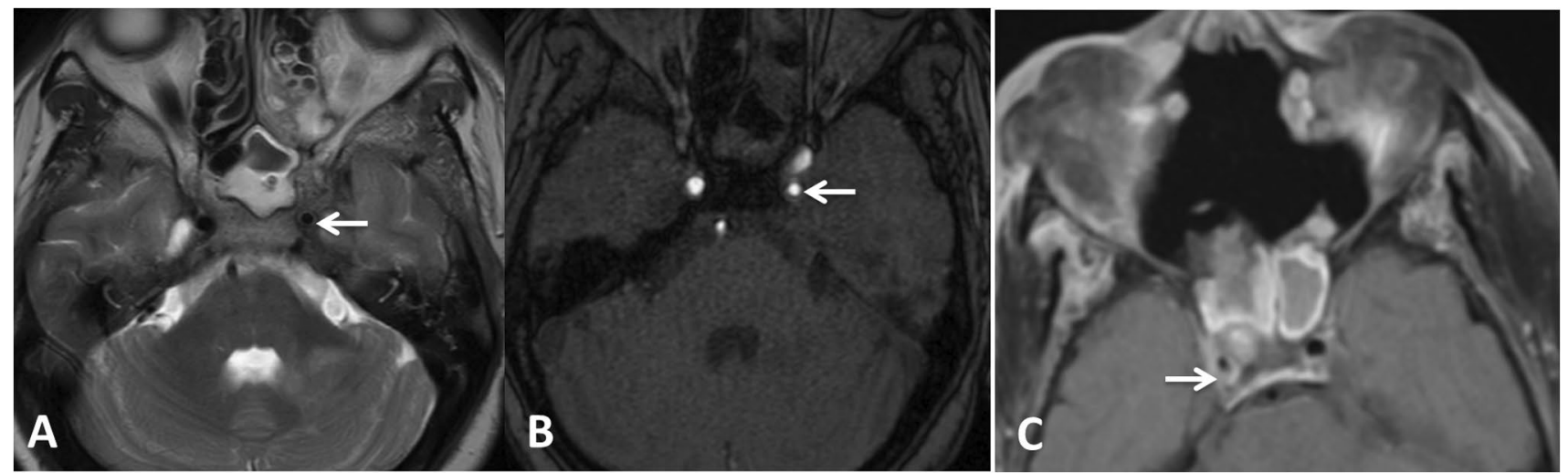

Fig. 3 Angioinvasion of wall of cavernous ICA: A Axial T2-weighted image reveals hyperintense thickening of the wall of the left cavernous ICA. Lumen is patent with maintained flow void. B Time of Flight intracranial MR Angiogram depicts the mild luminal narrow- ing of left cavernous ICA with maintained flow related enhancement. C Post contrast T1FS images show enhancement of the wall of the right cavernous ICA, which is patent but narrow calibred, as opposed to the normal left cavernous ICA infarcts in a known case of mucormycosis: A Diffusionweighted image and $\mathbf{B}$ apparent diffusion coefficient map reveal acute embolic infarcts in the right anterior centrum semiovale in the watershed territory, secondary to angioinvasive occlusion of the right ICA
Fig. 4 Watershed embolic
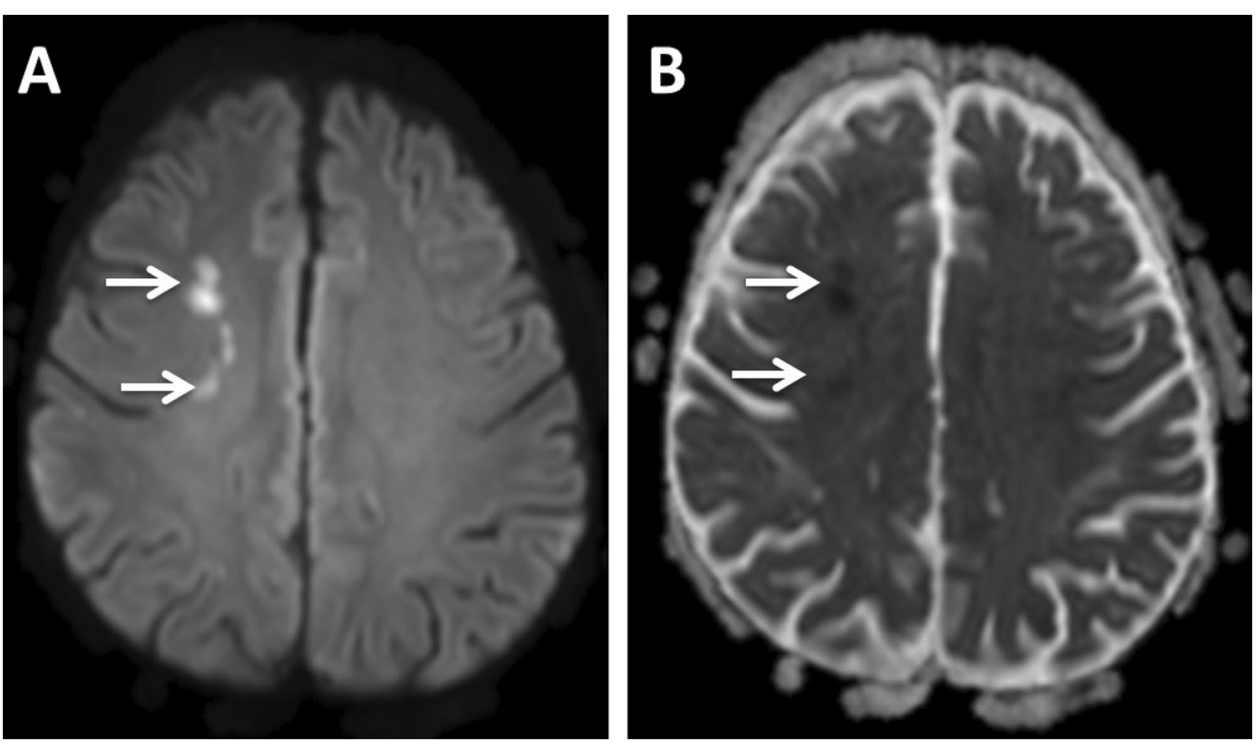

Fig. 5 Evolving vasculitic infarct in a known case of mucormycosis: A Diffusionweighted image and $\mathbf{B}$ apparent diffusion coefficient map reveal focal area of diffusion restriction in the right anterior gyrus rectus

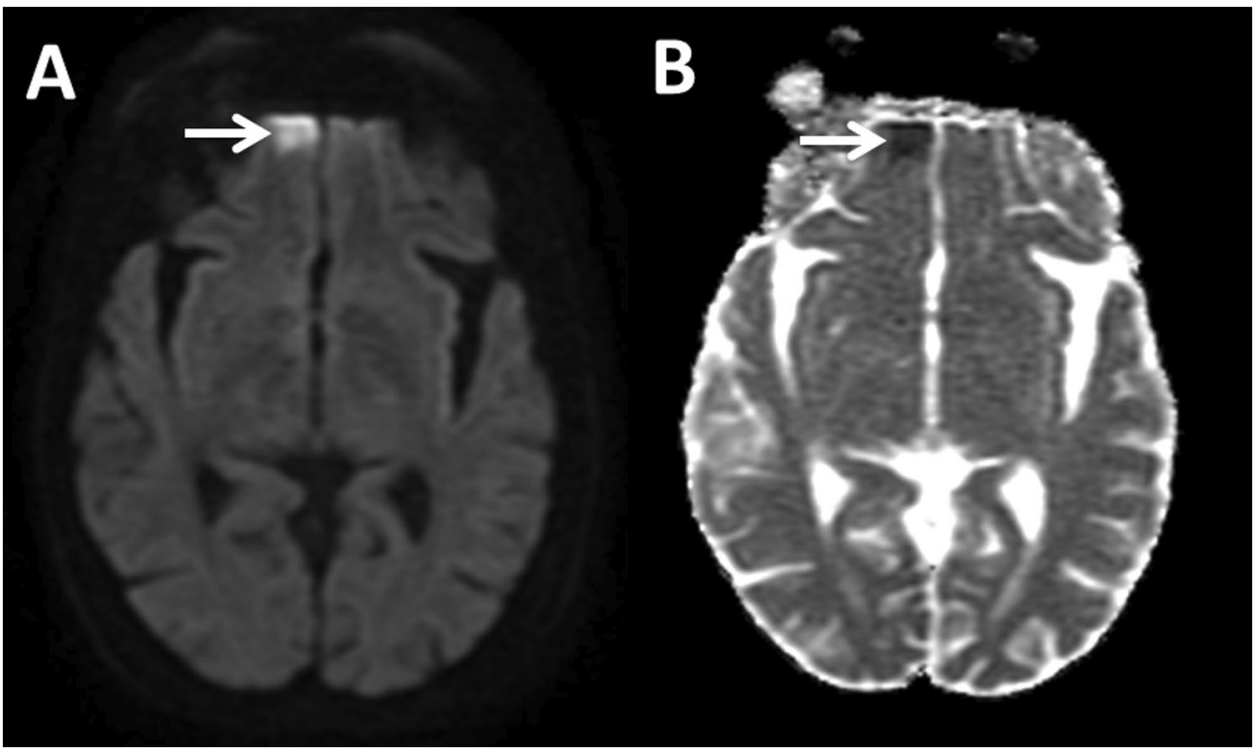


Fig. 6 Simultaneous infarction of bilateral optic nerves: A, B Axial DWI and ADC images show restricted diffusion in the intraorbital segments of bilateral and ADC maps reveal restricted diffusion in the right optic tract optic nerves. C, D Axial DWI
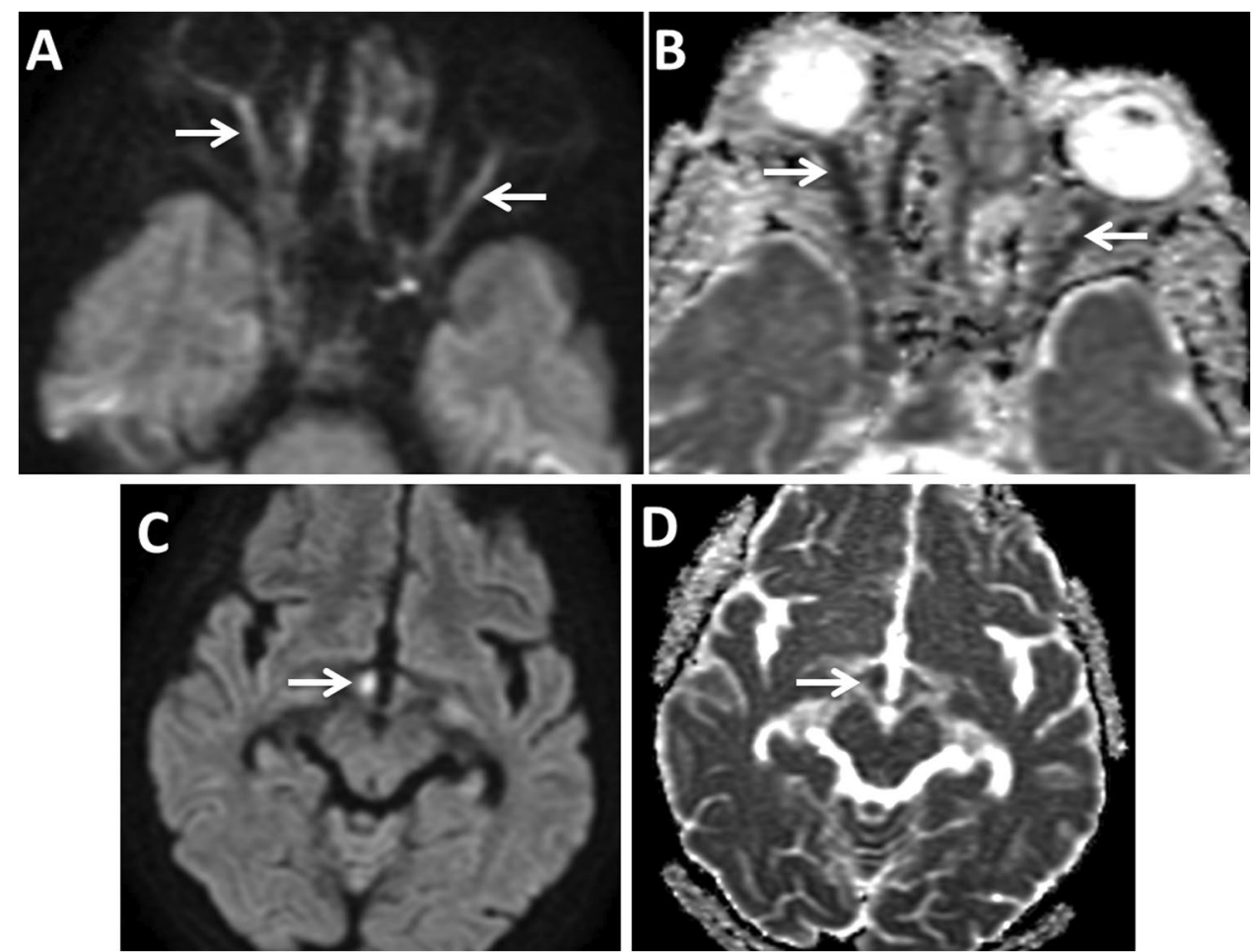

Fig. 7 Trigeminal nerve infarction and involvement of brainstem: A, B Restricted diffusion in the root entry zone, cisternal and gasserian segments of the left trigeminal nerve (white arrows). Right trigeminal nerve is normal. C Restricted diffusion in the right trigeminal nucleus. D Follow-up scan shows expansion of the FLAIR hyperintense infarct to involve the adjacent lateral surface of right hemipons
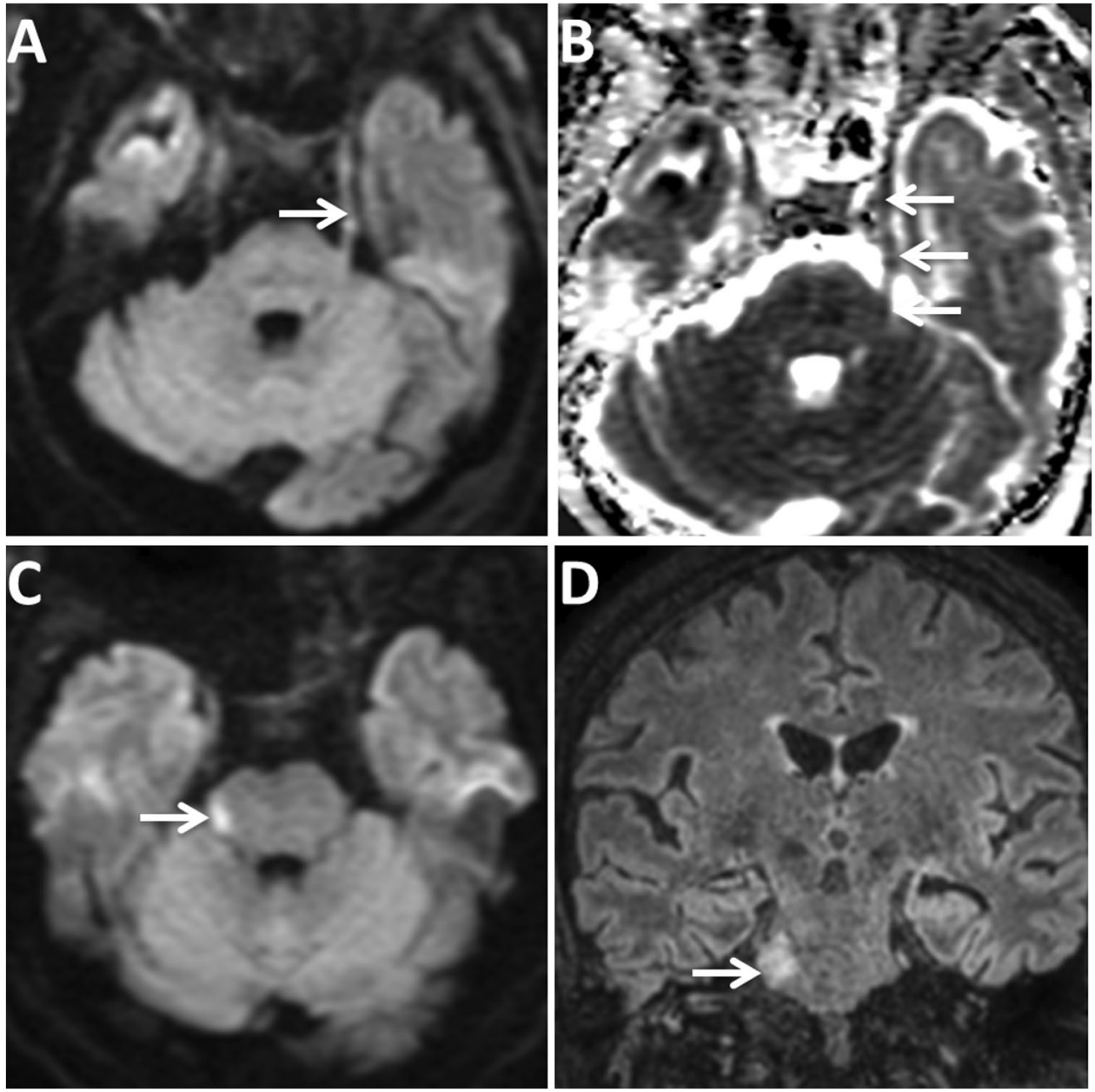
Fig. 8 Perineural spread of mucormycosis: Axial post contrast $\mathrm{T} 1$ fat suppressed images show A smooth enhancement of the cisternal segment of the right trigeminal nerve (arrow) and $\mathbf{B}$ spread along the branches of trigeminal branches through foramen ovale (arrow)
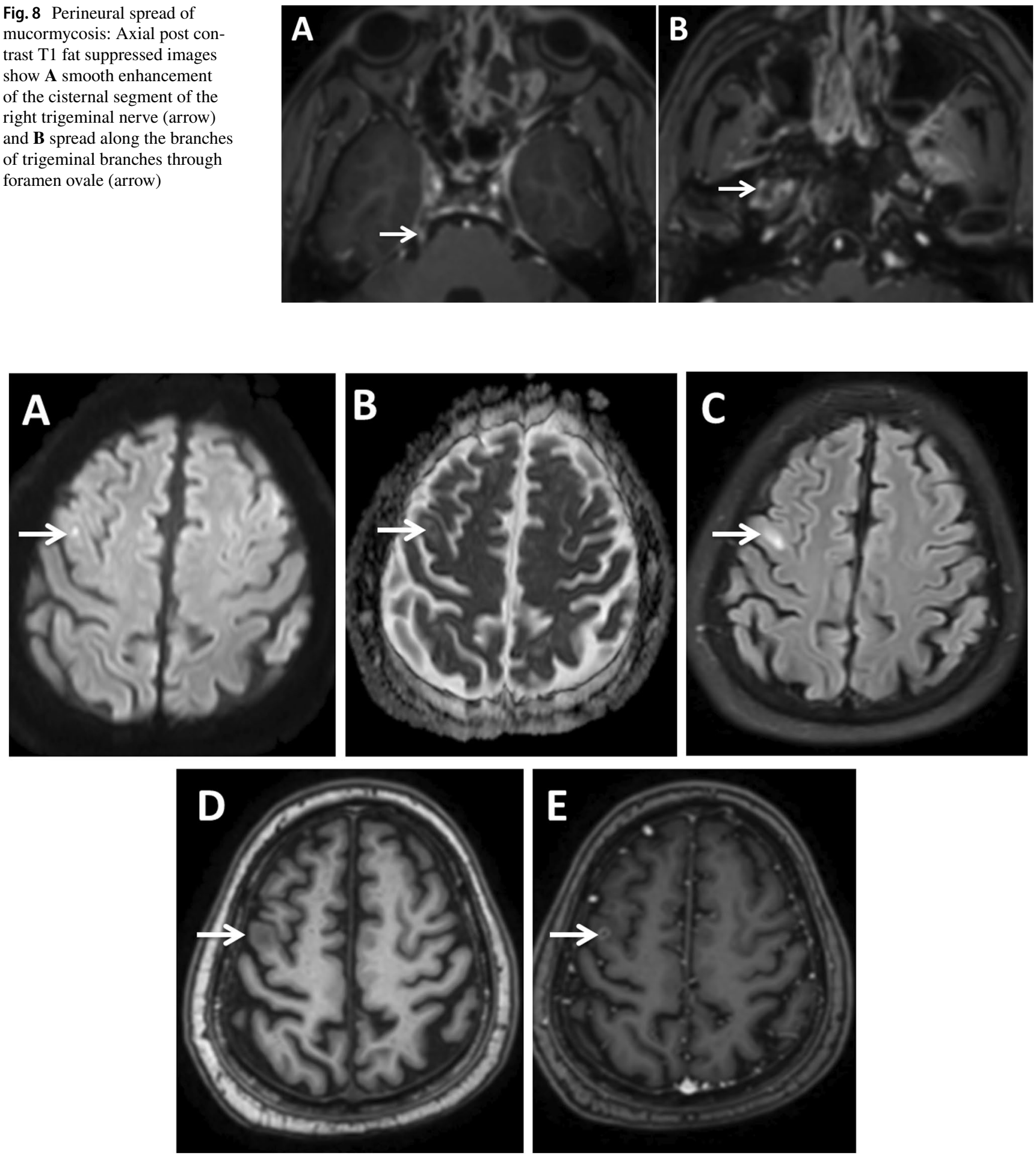

Fig. 9 Septic embolus in a known case of mucormycosis: A DWI, B ADC, C FLAIR, D axial T1WI, and $\mathbf{E}$ axial post contrast T1FS MRI images reveal a ring enhancing lesion in the right precentral sulcus with central restricted diffusion and mild surrounding oedema. No blooming was seen 

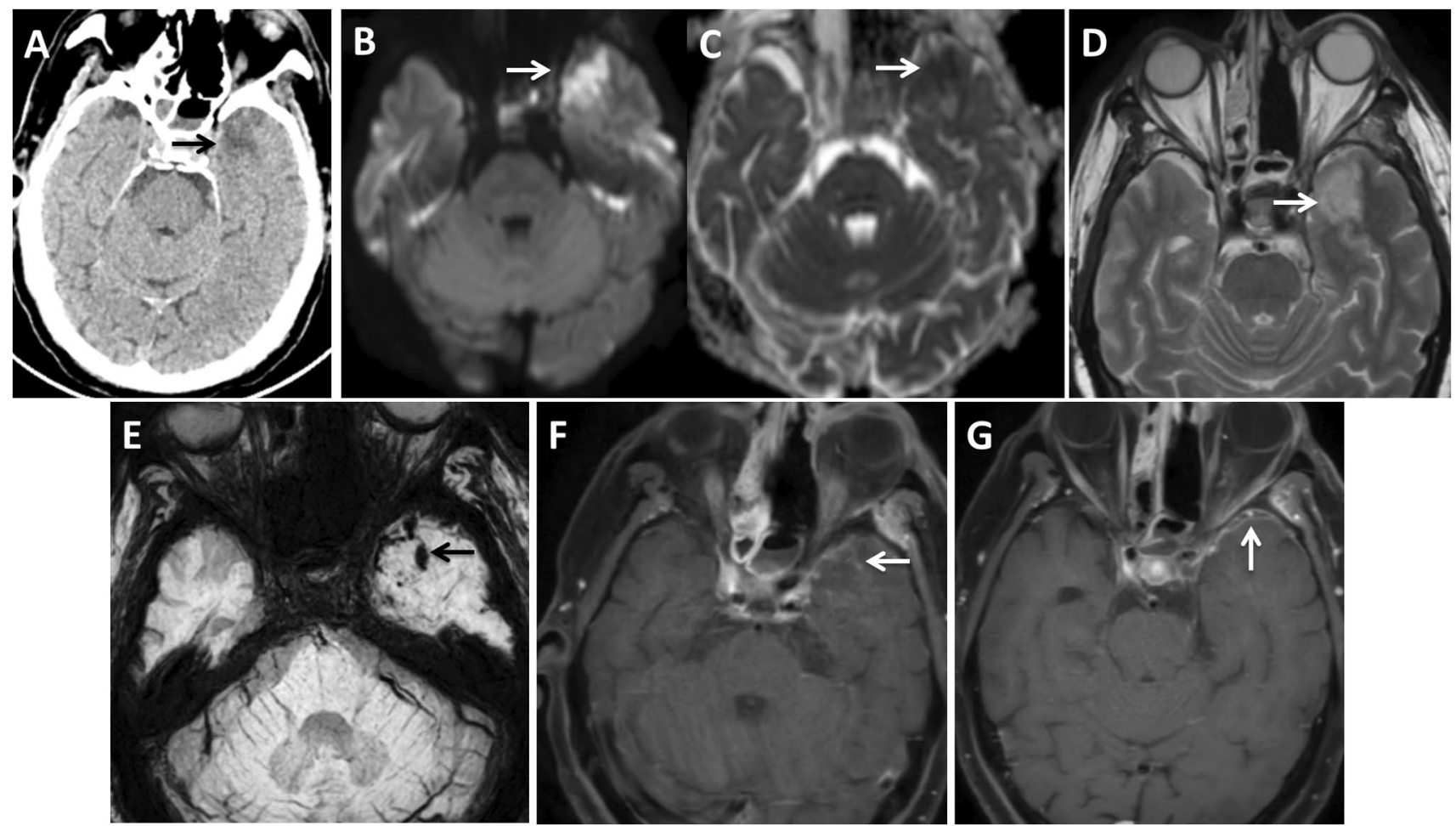

Fig. 10 Late cerebritis/evolving abscess: A Axial plain CT reveals illdefined hypodensity in the left temporal lobe. Axial plain and contrast MRI reveals ill-defined area of restricted diffusion $(\mathbf{B}, \mathbf{C}), \mathrm{T} 2$ hyperintensity (D), peripheral foci of blooming on SWI (E), and subtle

2). Associated dilatation and thrombosis of the superior ophthalmic vein may also be visualised.

\section{ICA involvement}

Angioinvasion of internal carotid artery across the sphenoid sinus or cavernous sinus and retrograde extension of mucor thrombus from the smaller ophthalmic arteries are the mechanisms involved. It can be evaluated on CT but is best evaluated on MRI. It presents as abnormal T2 signal intensity and post contrast enhancement in the wall (vasculitis) (Fig. 3), progressing to infiltration causing irregular luminal narrowing and ultimately complete thrombosis (Fig. 2) which is seen as loss of flow void on T2-weighted images [4].

\section{Infarction}

Involvement of the internal carotid artery can result in cerebral infarcts in the watershed territory, embolic in nature (Fig. 4). Infarcts can also occur secondary to direct thin enhancement in the lateral aspect of the lesion as seen on post contrast T1-weighted images $(\mathbf{F})$. G Adjacent thin pachymeningeal enhancement along the left temporal convexity

vessel invasion (Fig. 5), which then harbour the fungal elements, visualised as blooming within the infarct on susceptibility weighted images [4].

\section{Cranial nerve involvement}

Two unique characteristics of cranial nerve involvement by ROCM are described below:

- Ischaemic neuropathy. The optic nerve and the trigeminal nerve are the most commonly involved cranial nerves. Optic nerve infarction results from angioinvasion and thrombotic occlusion of the central retinal artery or ophthalmic artery or by direct infiltration of the nerve by the fungus. Trigeminal nerve infarction can also occur in a similar fashion. It is visualised as restricted diffusion along the affected nerve (Figs. 6 and 7), usually without any enlargement, altered T2/STIR signal intensity or post contrast enhancement $[10,11]$.

- Perineural spread. On MRI, direct findings suggestive of perineural spread are enlargement/thickening of the nerve, irregularity and excessive enhancement of the cranial nerve or its branch (within the cistern, canal, or 

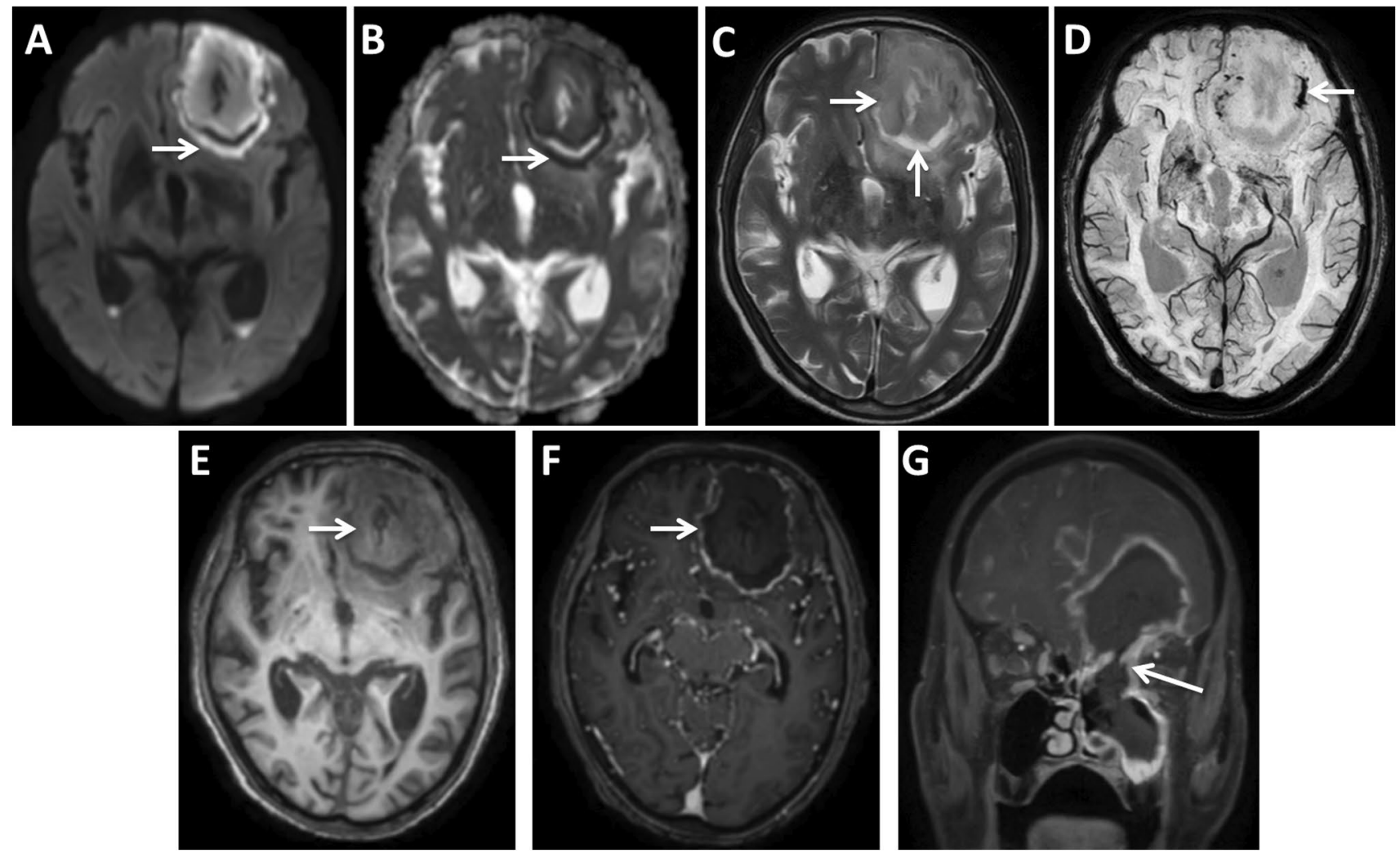

Fig. 11 Fungal cerebral abscess: (A) DWI, (B) ADC, (C) Axial T2WI, (D) SWI, (E) Axial T1WI and (F) Post contrast axial T1FS MRI images reveal an irregularly marginated, peripherally enhancing, $\mathrm{T} 2$ heterogeneously hyperintense and $\mathrm{T} 1$ isointense lesion in the left frontal lobe, showing peripheral diffusion restriction in the wall and

foramen) (Fig. 8), loss of the normal fat pad adjacent to the foramen, widening or excessive enhancement within the pterygopalatine fossa, Meckel's cave, or the cavernous sinus $[12,13]$. Perineural spread serves as a conduit of spread - for instance, from the orbit intracranially along the optic nerve, and along the trigeminal nerve to the brainstem (Fig. 7).

\section{Septic emboli}

Hyphal arterial invasion can result in septic emboli. They manifest as peripheral ring enhancing lesions, with central restricted diffusion and perilesional FLAIR hyperintense oedema, usually located at the grey matter - white matter junctions, predominantly in the middle cerebral artery territory (Fig. 9) [14]. Septic emboli may also be seen as multiple microhaemorrhages on Susceptibility weighted images [15]. foci of SWI blooming mainly on the left lateral aspect of the lesion. Perilesional oedema, mass effect and subfalcine herniation to the right side noted. (G) Post contrast coronal T1FS images depict communication of the abscess with the left frontal, ethmoid, and maxillary sinuses

\section{Cerebritis}

It presents on $\mathrm{CT}$ as ill-defined intraparenchymal hypodense lesions with scattered areas of increased attenuation due to either haemorrhage or increased concentration of metal ions. On MRI, early cerebritis appears as a cortical-subcortical T1 iso-hypointense, T2/FLAIR hyperintense area with patchy restricted diffusion, and no post contrast enhancement. Progressive peripheral enhancement may be seen in late cerebritis (Fig. 10) [16].

\section{Abscess}

Cerebral parenchymal abscess due to Mucor are most often seen in the frontal lobe or temporal lobes. They appear as irregularly marginated $\mathrm{T} 2$ hyperintense lesions with $\mathrm{T} 2$ hypointense, irregular peripherally enhancing walls with intracavitary non-enhancing projections with characteristic restricted diffusion of the intracavitary projections and of the wall. Susceptibility artefacts may be seen in the periphery of the abscess indicating a fungal composition 
Fig. 12 Leptomeningitis and ventriculitis in a case of mucormycosis post FESS: Axial post contrast FLAIR images reveal diffuse post contrast enhancement of bilateral hemispheric sulcal spaces (A, B) (white arrows), basal cisterns cisterns (C), and the cerebellar foliae (D). B Post contrast enhancement of the ependymal lining, ventricular walls (yellow arrows), hydrocephalus with dependent exudates in the occipital horns of bilateral lateral ventricles (arrow heads)
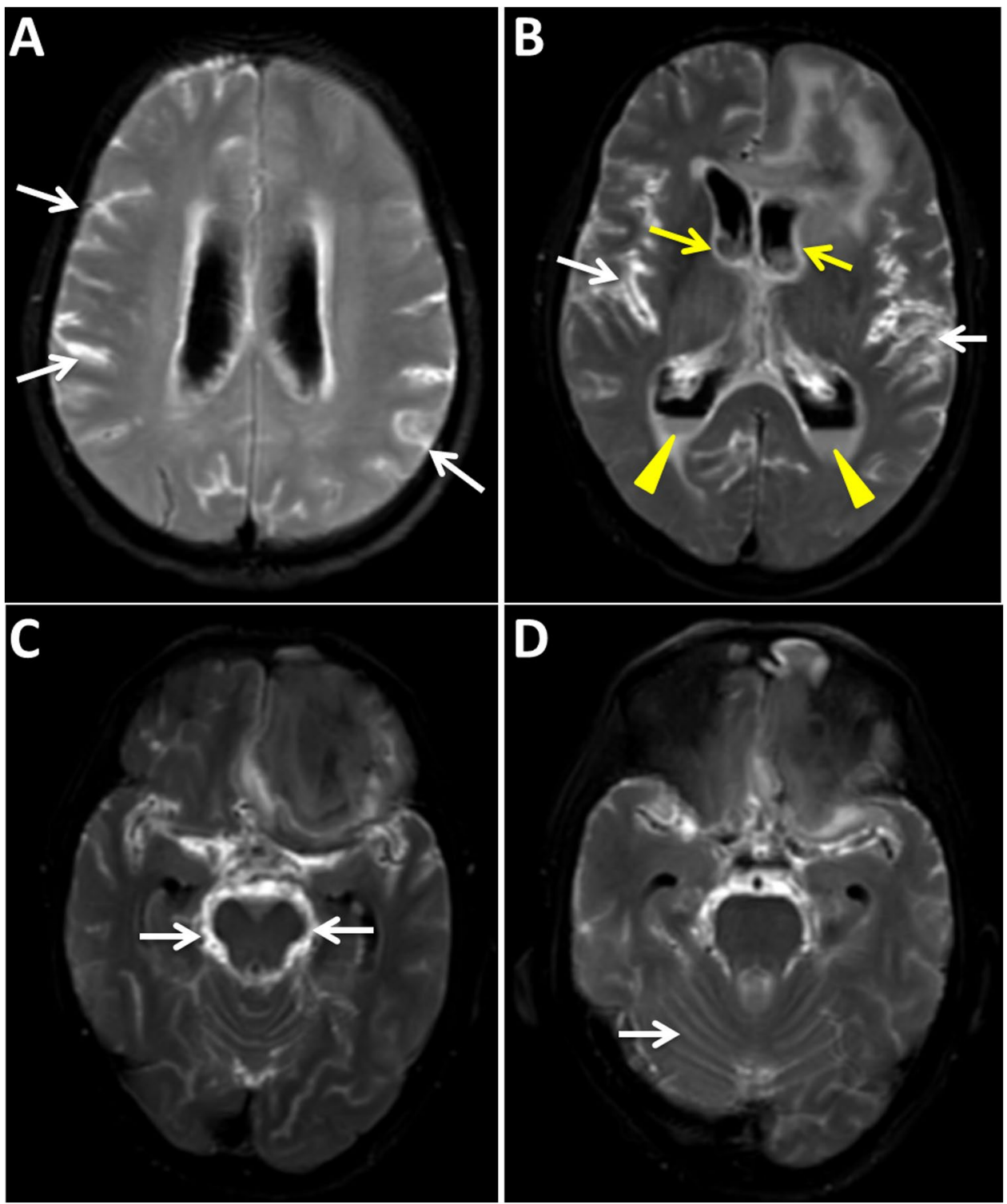
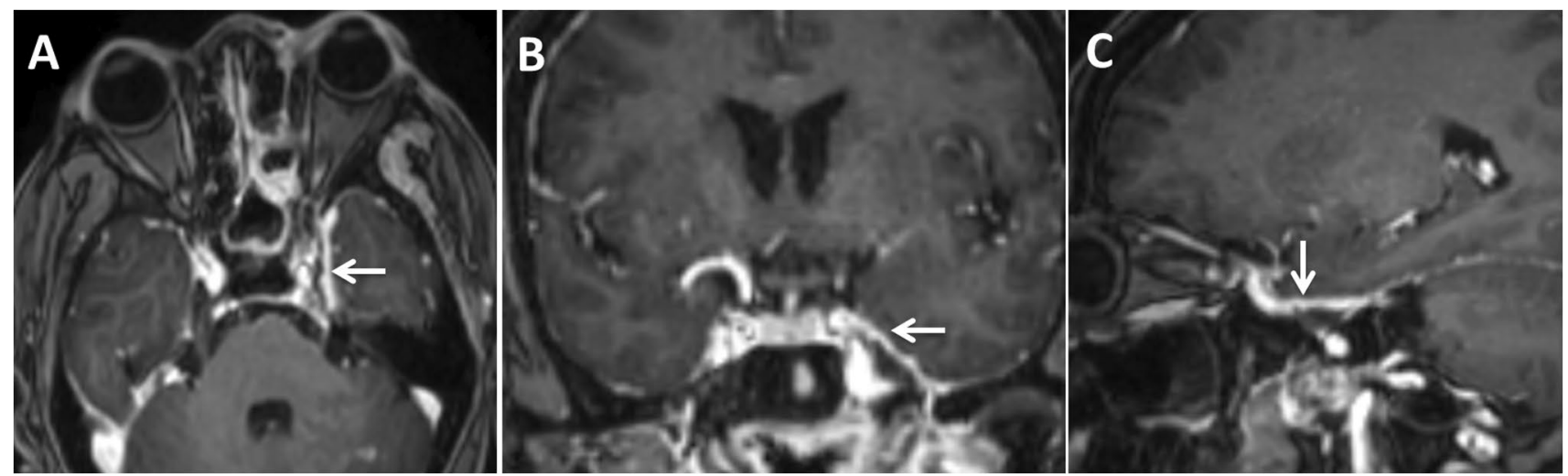

Fig. 13 Pachymeningitis: Post contrast T1 weighted fat suppressed images in $\mathbf{A}$ axial, $\mathbf{B}$ coronal, and $\mathbf{C}$ sagittal planes demonstrate pachymeningeal enhancement along the medial aspect of left temporal lobe and floor of middle cranial fossa 
Fig. 14 Direct extension across ethmoid: In a case with known mucormycosis post FESS, A coronal post contrast T1 fat suppressed images show dural enhancement along the crista galli and base of anterior cranial fossa, indicating direct intracranial spread across the ethmoid sinus. B Coronal T2 fat suppressed image shows $\mathrm{T} 2$ hyperintense oedema in the right frontal lobe with no focal lesion
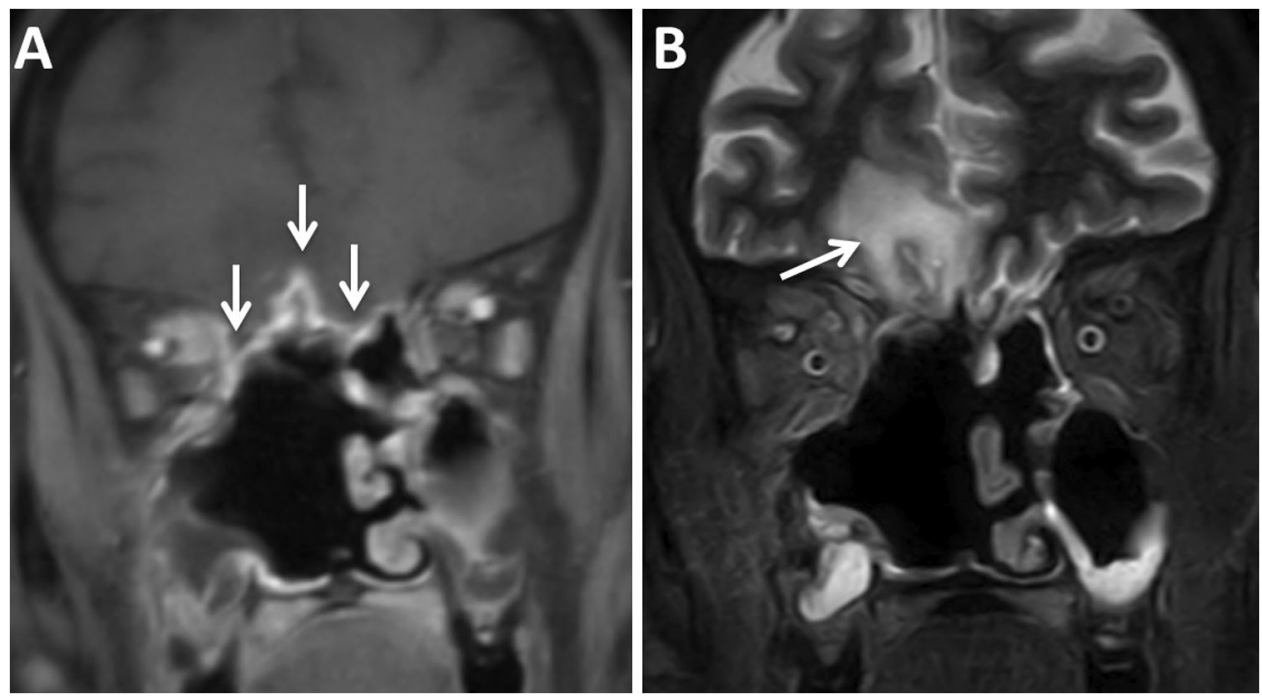

Fig. 15 Base of skull involvement: Patchy STIR hyperintensity in the A right pterygoid and $\mathbf{B}$ clivus, represents marrow oedema
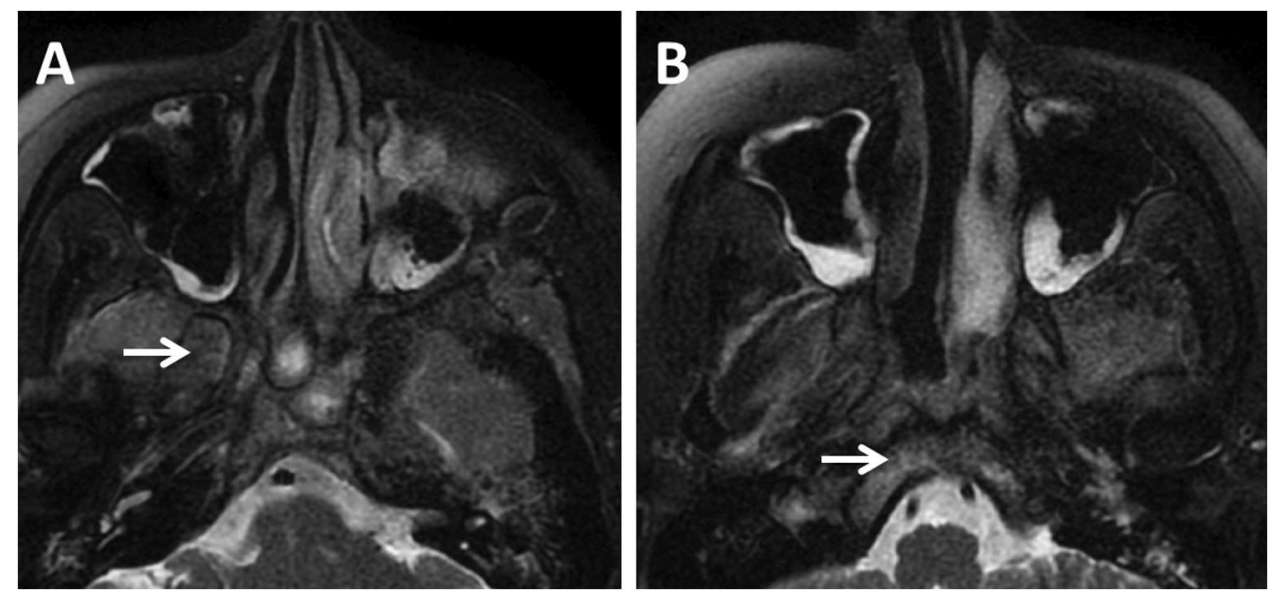

(Fig. 11) [10]. Ventriculitis (Fig. 12) and subdural/extradural abscesses are rare may be seen in advanced disease.

\section{Haemorrhage}

Intracerebral haematoma is a rare manifestation of ROCM, seen as a result of possible ruptured mycotic aneurysm which are most often seen in the anterior circulation $[9,16]$

\section{Leptomeningitis/pachymeningitis}

MR images demonstrate thick, often nodular, meningeal enhancement on post contrast T1-weighted and FLAIR images, due to thick gelatinous exudates containing inflammatory cells, fibrin, and haemorrhage leading to arachnoiditis which in turn can lead to secondary hydrocephalus (Fig. 12) [16]. Dural thickening and enhancement (Figs. 13 and 14) is commonly in seen in the middle cranial fossa and paracavernous dura as well as along the frontal convexities and, rarely, can be diffuse.

\section{Skull base involvement}

Being an angioinvasive fungus, bony involvement is not common and is usually seen only in chronic forms of the disease. However, early involvement of the bone marrow can be picked up on MRI, seen as patchy STIR hyperintense marrow oedema (Fig. 15) and heterogeneous post contrast enhancement [17]. Chronic forms can be visualised on CT as rarefaction, osteolysis, erosions, and sclerosis [18].

\section{Conclusion}

An exponential increase in the incidence of ROCM was witnessed in the second wave of the COVID-19 pandemic in India, reiterating the importance of familiarising oneself with its varied but crucial manifestations on imaging. MRI with contrast forms the crux of patient management and the 
brain has to be imaged in every case. It aids in diagnosis, assessment of extent of disease, and early identification of intracranial extension which may contribute to the reduction of mortality as it allows for timely initiation of aggressive treatment.

Funding This study was not supported by any funding.

Data availability Not applicable.

Code availability Not applicable.

\section{Declarations}

Conflicts of interest The authors declare that they have no conflict of interest.

\section{References}

1. Singh A, Singh R, Joshi S, Misra A (2021) Mucormycosis in COVID-19: a systematic review of cases reported worldwide and in India. Diabetes Metab Syndr 15:102146. https://doi.org/10. 1016/j.dsx.2021.05.019

2. Roden MM, Zaoutis TE, Buchanan WL, Knudsen TA, Sarkisova TA, Schaufele RL et al (2005) Epidemiology and outcome of zygomycosis: a review of 929 reported cases. Clin Infect Dis 41:634-653. https://doi.org/10.1086/432579

3. Herrera DA, Dublin AB, Ormsby EL, Aminpour S, Howell LP (2009) Imaging findings of rhinocerebral mucormycosis. Skull Base 19:117-125. https://doi.org/10.1055/s-0028-1096209

4. Patil A, Mohanty HS, Kumar S, Nandikoor S, Meganathan P (2016) Angioinvasive rhinocerebral mucormycosis with complete unilateral thrombosis of internal carotid artery-case report and review of literature. BJR Case Rep 2:20150448. https://doi.org/ 10.1259/bjrcr.20150448

5. Gamaletsou MN, Sipsas NV, Roilides E, Walsh TJ (2012) Rhinoorbital-cerebral mucormycosis. Curr Infect Dis Rep 14:423-434. https://doi.org/10.1007/s11908-012-0272-6

6. Gavito-Higuera J, Mullins CB, Ramos-Duran L, Olivas Chacon CI, Hakim N, Palacios E (2016) Fungal infections of the central nervous system: a pictorial review. J Clin Imaging Sci 6:24. https://doi.org/10.4103/2156-7514.184244
7. Mathur S, Karimi A, Mafee MF (2007) Acute optic nerve infarction demonstrated by diffusion-weighted imaging in a case of rhinocerebral mucormycosis. AJNR Am J Neuroradiol 28:489-490

8. Ghuman MS, Kaur S, Bhandal SK, Ahluwalia A, Saggar K (2015) Bilateral optic nerve infarction in rhino-cerebral mucormycosis: a rare magnetic resonance imaging finding. J Neurosci Rural Pract 6:403-404. https://doi.org/10.4103/0976-3147.158790

9. Lone PA, Wani NA, Jehangir M (2015) Rhino-orbito-cerebral mucormycosis: magnetic resonance imaging. Indian J Otol 21:215-218. https://doi.org/10.4103/0971-7749.159700

10 Chikley A, Ben-Ami R, Kontoyiannis DP (2019) Mucormycosis of the central nervous system. J Fungi (Basel) 5:59. https://doi. org/10.3390/jof5030059

11. Bhatia H, Kaur R, Bedi R (2020) MR imaging of cavernous sinus thrombosis. Eur J Radiol Open 7:100226. https://doi.org/10. 1016/j.ejro.2020.100226

12. Koc Z, Koc F, Yerdelen D, Ozdogu H (2007) Rhino-orbital-cerebral mucormycosis with different cerebral involvements: infarct, hemorrhage, and ophthalmoplegia. Int J Neurosci 117:1677-1690. https://doi.org/10.1080/00207450601050238

13. Champey J, Pavese P, Bouvaist H, Kastler A, Krainik A, Francois $P$ (2016) Value of brain MRI in infective endocarditis: a narrative literature review. Eur J Clin Microbiol Infect Dis 35:159-168. https://doi.org/10.1007/s10096-015-2523-6

14. Scully MA, Yeaney GA, Compton ML, Berg MJ (2012) SWAN MRI revealing multiple microhemorrhages secondary to septic emboli from mucormycosis. Neurology 79:1932-1933. https:// doi.org/10.1212/wnl.0b013e318271f86c

15. Orguc S, Yücetürk AV, Demir MA, Goktan C (2005) Rhinocerebral mucormycosis: perineural spread via the trigeminal nerve. J Clin Neurosci 12:484-486. https://doi.org/10.1016/j.jocn.2004. 07.015

16. Parsi K, Itgampalli RK, Vittal R, Kumar A (2013) Perineural spread of rhino-orbitocerebral mucormycosis caused by Apophysomyces elegans. Ann Indian Acad Neurol 16:414-417. https://doi.org/10.4103/0972-2327.116921

17. Chan LL, Singh S, Jones D, Diaz EM Jr, Ginsberg LE (2000) Imaging of mucormycosis skull base osteomyelitis. AJNR Am J Neuroradiol 21:828-831

18. Therakathu J, Prabhu S, Irodi A, Sudhakar SV, Yadav CK, Rupa V (2018) Imaging features of rhinocerebral mucormycosis: a study of 43 patients. Egypt J Radio Nucl Med 49:447-452. https://doi. org/10.1016/j.ejrnm.2018.01.001

Publisher's note Springer Nature remains neutral with regard to jurisdictional claims in published maps and institutional affiliations. 